\title{
Sistemas integrados de gestión en el sector minero*
}

[Artículos de revisión]

\author{
Zulma Rocío Gil Albarracín** \\ Ingrid Carolina Moreno Rodríguez ${ }^{* * *}$
}

Recibido: 11 de noviembre de 2020

Revisado: 25 de enero de 2021

Aceptado: 13 de marzo de 2021

Citar como:

Gil Albarracín, Z. R. y Moreno Rodríguez I. C. (2021). Sistemas integrados en el sector minero. Signos, Investigación en Sistemas de Gestión, 13(2).

https://doi.org/10.15332/24631140.6674

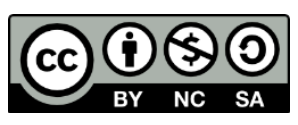

\section{Resumen}

Los sistemas de gestión se han convertido en facilitadores para el desarrollo y logro de los objetivos corporativos trazados por las organizaciones, aumentan el rendimiento, identifican problemas críticos y dan vertientes para su solución, aportan en la administración y gestión de los recursos, generan la posibilidad de contar con estándares que facilitan el ingreso a nuevos mercados y el control de

\footnotetext{
* Artículo de revisión.

** Especialista es Seguridad y Salud en el Trabajo e ingeniera industrial por la Universidad Santo Tomás, Bogotá, Colombia. Correo electrónico: zulmagil.anat@gmail.com, zulmagil@usantotomas.edu.co; ORCID: https://orcid.org/0000-0002-6570-9382; CvLAC:

https://scienti.minciencias.gov.co/cvlac/visualizador/generarCurriculoCv.do?cod rh $=0001776057$ *** Magíster en Gestión Documental y Administración de Archivos, especialista en Administración y Gerencia de Sistemas de Calidad y administradora de empresas por la Universidad Santo Tomás, Bogotá, Colombia. Correo electrónico: ingridcmoreno@usantotomas.edu.co, cmrcalidad21@gmail.com; ORCID https://orcid.org/0000-0003-3624-0877. CvLAC: http://scienti.colciencias.gov.co:8081/cvlac/visualizador/generarCurriculoCv.do?cod rh $=0000101$ $\underline{575}$
}

Signos, Investigación en Sistemas de Gestión

ISSN: 2145-1389 | e-ISSN: 2463-1140 | DOI: https://doi.org/10.15332/24631140

Vol. 13 N.o 2 | julio-diciembre de 2021 
forma estratégica en la respuesta a las necesidades de las partes interesadas y de un contexto que cada vez presenta mayores desafíos. Para el sector minero, representan la posibilidad de gestionar de manera integrada componentes que impactan favorablemente en los resultados trazados, en la protección de sus trabajadores y en el manejo de buenas prácticas desde el componente ambiental. El objetivo del presente artículo es identificar, desde una revisión bibliográfica, los beneficios de integrar el sistema de gestión de calidad, el sistema de gestión ambiental y el sistema de gestión de seguridad y salud en el trabajo en el sector minero, a partir de la consulta en las bases de datos Science Direct, Taylor Francis Online, Springer link, Web of Sciencie, Pumbed. Los resultados obtenidos evidenciaron que las intervenciones, a través de la ejecución de los sistemas de gestión, logran mejorar los procesos de la organización y se resalta que el liderazgo por parte de la alta gerencia juega un papel vital y significativo en el trabajo y en el cumplimiento de los objetivos trazados. La mayoría de las organizaciones buscan que los sistemas de gestión solucionen sus problemas internos como la accidentalidad, la estandarización, el mejor aprovechamiento del tiempo; además de implementar acciones encaminadas a la estandarización de procesos, el cumplimiento de metas y el logro sistémico de la mejora continua.

Palabras clave: sector minero, sistema de gestión de calidad, sistema de gestión ambiental, sistema de gestión de seguridad y salud en el trabajo, sistemas integrados de gestión.

\section{Integrated management systems in the mining sector}

\section{Abstract}

Management systems have become facilitators for the development and achievement of corporate objectives set by organizations, increase performance, identify critical problems and provide guidelines for their solution, contribute to resources administration and management, 
generate the possibility to develop standards that facilitate entry into new markets and control strategically in response to stakeholders needs and a context that increasingly poses greater challenges. For the mining sector, they provide the possibility of comprehensively managing components that impact positively on the results outlined, on the protection of its workers and on the management of good practices from the environmental component. This article objective is to identify, based on a literature review, the benefits of integrating the quality management system, the environmental management system and the occupational health and safety management system in the mining sector, based on the query of databases such as Science Direct, Taylor Francis Online, Springer link, Web of Science, and Pumbed. The results obtained showed that the interventions, through the management systems, manage to improve the organization processes and it is highlighted that the leadership by the top management plays a vital and significant role in the work and in the fulfillment of the objectives set. Most of the organizations look for the management systems to solve their internal problems such as accidents, standardization, the best use of time, in addition to implementing actions aimed at processes standardization, goals fulfillment and the systemic achievement of continuous improvement.

Keywords: mining sector, quality management system, environmental management system, occupational health and safety management system, integrated management systems.

\section{Sistemas integrados de gestão no setor de mineração}

\section{Resumo}

Os sistemas de gestão se tornaram facilitadores para o desenvolvimento e a realização dos objetivos corporativos estabelecidos pelas organizações, aumentam o desempenho, identificam problemas críticos e fornecem orientações para sua solução, contribuem para a administração e gestão

Signos, Investigación en Sistemas de Gestión

ISSN: 2145-1389 | e-ISSN: 2463-1140 | DOI: https://doi.org/10.15332/24631140

Vol. 13 N. ${ }^{\circ} 2$ | julio-diciembre de 2021 
de recursos, geram a possibilidade de desenvolver padrões que facilitam a entrada em novos mercados e controlam estrategicamente em resposta às necessidades das partes interessadas e um contexto que cada vez mais coloca maiores desafios. Para o setor de mineração, elas representam a possibilidade de gerenciar integradamente componentes que têm um impacto favorável sobre os objetivos estabelecidos, na proteção de seus trabalhadores e na gestão de boas práticas do componente ambiental. Este artigo visa identificar, com base em uma revisão bibliográfica, os benefícios de integrar o sistema de gestão da qualidade, o sistema de gestão ambiental e o sistema de gestão da saúde e segurança ocupacional no setor de mineração, partindo da consulta nas bases de dados Science Direct, Taylor Francis Online, Springer link, Web of Sciencie e Pumbed. Os resultados obtidos mostraram que as intervenções, através da implementação de sistemas de gestão, conseguem melhorar os processos da organização e destaca-se que a liderança da alta administração tem um papel vital e significativo no trabalho e no atingimento dos objetivos estabelecidos. A maioria das organizações busca que sistemas de gestão resolvam seus problemas internos, tais como acidentes, padronização, melhor aproveitamento do tempo, além de implementar ações voltadas a padronizar processos, cumprir metas e alcançar uma melhoria contínua sistêmica.

Palavras-chave: setor de mineração, sistema de gestão de qualidade, sistema de gestão ambiental, sistema de gestão de saúde e segurança ocupacional, sistemas de gestão integrada.

\section{Introducción}

La minería ha sido una de las actividades fundamentales para el progreso económico del país, ya que la mayoría de los bienes materiales provienen de la transformación de productos naturales, en especial los recursos mineros; sin embargo, esta es conocida también por los desastres a gran escala que han cobrado la vida de muchos trabajadores. Actualmente, la investigación de intensidad laboral en minería de carbón (Boal, 2018) hace 
un análisis entre producción vs. seguridad del minero y su conclusión es que el minero arriesga su vida por generar más producción y ganar más dinero, acostumbrándose al riesgo sin imaginar que puede más adelante cobrarle la vida.

La actividad minera en los últimos años en el país ha aumentado de manera exagerada y consigo el nivel de accidentalidad, ya que la minería subterránea presenta unas características especiales que la hacen mucho más riesgosa al tratarse de construcciones de roca natural, de trabajo en espacios confinados, con generación de gases, derrumbes, entre otros peligros. El sector minero enfrenta desafíos, como lo menciona Domingues et ál. (2017), debido a factores internos inesperados (falta de personal capacitado o fallas frecuentes de los equipos) y factores externos (precios de las materias primas, minerales, volatilidad del mercado, regulaciones crecientes, ganancias menguantes y demanda global cambiante) pero aun así aporta ingresos al PIB de cada país y genera progreso.

Una gestión de riesgos inadecuada puede provocar fallos en la producción $\mathrm{y}$, en ocasiones, lesiones graves al personal y problemas ambientales, tales problemas pueden interrumpir proyectos e incluso llevar a la pérdida total del negocio (Bayram, 2020).

El desarrollo de un buen sistema de gestión logra reducir escenarios como los identificado por Bayram en su artículo. Al respecto, Vanek et ál. (2015) identifican que la metodología Kaizen es la correcta para aplicar en una organización minera por su facilidad, ya que logra involucrar a los trabajadores con métodos como tarjetas de ideas (sugerencias), educación a los trabajadores, entre otras. Esto ayuda a mejorar las condiciones de trabajo y ambiente, producción, medidas y mejoras en toda la compañía, todo esto funcionara especialmente si una empresa quiere mantener su posición en el mercado, pues logra apoyar a la toma eficiente de 
decisiones, logro oportuno de objetivos corporativos y así como el cumplimiento de los requisitos legales aplicables al sector.

Actualmente, el sector minero ocupa uno de los índices de accidentalidad de más alto riesgo para la salud de los trabajadores, las empresas deben implementar normas y reglamentos de gestión con el fin de garantizar la concienciación del trabajador y la importancia que tiene para la organización. Estas empresas se enfrentan a un sector minero de rápido crecimiento y hace necesario conocer los factores influyentes para reducir las muertes relacionadas con el trabajo y aumentar la eficiencia de la empresa. Una cultura de seguridad madura, como lo menciona Stemn et ál. (2019), se considera un medio importante para garantizar un buen desempeño en materia de seguridad, especialmente en la reducción de accidentes; pero llegar a un nivel de madurez fuerte debe ir de la mano con la implementación de un sistema de gestión estructurado, que garantice un nivel importante de conciencia frente a la seguridad.

El desarrollo de los sistemas de gestión para el sector minero es un campo complejo, como lo identifico Pantoja Timarán y Pantoja Barrios (2016), pues el ambiente adverso y las percepción de riegos son diferente entre los mineros; por esto, desde el desarrollo del presente artículo, se determina cómo el desarrollo de los sistemas de gestión impacta al sector de manera positiva, y cómo podría relacionarse para la mediana minería, los cuales, según la Agencia Nacional de Minería, ocupan actualmente un gran porcentaje en títulos mineros de explotación en el país (Agencia Nacional de Minería, 2020a).

El sistema de gestión de seguridad y salud en el trabajo para el sector minero es muy amplio, ya que la legislación Colombia aporta un gran número de reglamentaciones articuladas al proceso de la minería; por ejemplo, el Decreto 1072 de 2015 (Decreto Único Reglamentario del Sector 
Trabajo) y el Decreto 1886 de 2015, los cuales refuerzan la ejecución de la minería en el ejercicio propio de sus actividades y la seguridad y salud en el trabajo. Dentro del análisis bibliográfico, se encuentra que el primero deber en los procesos de minera, dentro del sistema de gestión de seguridad y salud en el trabajo, es identificar los riesgos (Samantra et ál., 2017), para esto se contemplan 3 factores: las consecuencias, el periodo de exposición y la probabilidad. Según el resultado de la evaluación de riesgos, se deben tomar medidas para eliminar los riesgos, preferiblemente, en la primera instancia.

Para los procesos de minería, de acuerdo con Pink et ál. (2016), llevar a cabo estos estándares es esencial, así mismo la implementación de medidas de control, pues es la única alternativa que se tiene. Por esto, es importante saber hacer la evaluación de peligros, ya que de esto depende que los controles sean eficaces. Para Chen y Zorigt (2013), uno de los objetivos del sistema de gestión de seguridad y salud en el trabajo es ayudar al gerente a comprender cómo se gestionan adecuadamente los riesgos para la salud y la seguridad y atenuar el impacto negativo de estos sobre la población trabajadora. Como lo menciona Stewa (2019), es importante trabajar en la reducción de peligros para preservar la salud de los trabajadores, esto basado en una buena identificación.

A nivel mundial hay países más avanzados en la explotación de carbón y su seguridad; por ejemplo, en China se ha hecho un ejercicio de identificación de peligros y lo han distribuido en 3 capas de defensa, es decir, cuando un peligro llega a la tercera capa este se materializa y se convierte en un accidente como lo menciona Liu et ál. (2020). Lo importante es no llegar a esta última capa.

Dentro de las etapas defensa identificas, se contemplan 6 pasos convencionales para el desarrollo de un sistema de gestión de seguridad, 
los cuales son la toma de decisiones, la preparación de liderazgo, la planificación y diseño de sistemas, la identificación y tratamiento de peligros, y la auditoría interna. Para lograr que estos pasos sean exitosos, se requiere un complemento a través de una cultura de seguridad, una cultura institucional, una cultura conceptual, una cultura material y una cultura de comportamiento. Todo esto logra que los sistemas de gestión sean eficientes y prosperen dentro de la organización bajo la participación de los trabajadores.

Si se compara a Colombia con China, aquí se tienen métodos de identificación de peligros como la Guía técnica colombiana GTC 45 (Icontec, 2010), la cual ayuda a priorizar los peligros más altos y poder generar controles oportunos para que no se materialicen. Sin embargo, se debe aprender más de las prácticas chinas que van más allá de un mejoramiento continuo hacia una transformación en procesos y cultura. En Europa y Asia se hace un enfoque fuerte en la cultura comportamental del minero, en quien comienza el hábito de transformación y adaptación. En Colombia la mayoría de los controles se basan en el uso de elementos de protección personal para el trabajador y se deja de lado la cultura; lograr superar esta mentalidad es un gran reto para el área de seguridad y salud en el trabajo.

En el contexto de la minería en Colombia, se identifica que en el año 2019 se tuvo una reducción de fatalidades en el sector minero (subterráneo). Se presentaron 81 fatalidades en todo el 2019 y los principales peligros fueron: derrumbe, atmósferas contaminadas y explosión. Sin embargo, en el primer semestre del 2020 las cifras fueron alarmantes, se han presentado 80 fatalidades, igualando la cifra que se tuvo en todo el 2019, y los principales peligros fueron: explosiones mineras $30 \%$, atmósferas contaminadas $27.5 \%$, derrumbes $15 \%$ y otros $10 \%$. De acuerdo con la 
información que publica la autoridad minera colombiana (Agencia Nacional de Minería, 2020b)

Dentro de los estudios que se han llevado a cabo sobre la accidentalidad en la minería se encuentra el realizado por Mirzaei Aliabadi et ál. (2018), quienes identifican que sin el compromiso de la dirección es poco probable que los programas de seguridad tengan éxito y que un buen clima organizacional es la causa fundamental del desempeño deficiente o eficiente en la seguridad de la organización. Esto deja claro que, para reducir la tasa de accidentes mineros, se deben abordar las deficiencias en los niveles organizativos superiores, es decir, la responsabilidad de los niveles superiores no la puede asumir un operativo.

El sistema de gestión de la calidad en el sector minero cuenta con un enfoque particular que dista en gran manera de otros sistemas, pues apuesta al mejoramiento de sus procesos, a la satisfacción de los clientes y a la estandarización de estos, con el fin de entregar productos o servicios únicos y confiables al consumidor (Marchiori y Mendes, 2020). El sistema de gestión de la calidad logra que la organización establezca la gestión de los procesos, como lo identifican Sadeghi Moghadam et ál. (2019), y dé paso a diversas herramientas, técnicas, principios y modelos con el fin de gestionar la calidad, la cual logra la adaptación de la población trabajadora de manera positiva para el cumplimiento de metas.

En el sector minero, las grandes empresas se adaptan a estos estándares y las que aún están en esa transición son las de pequeña y mediana minería, aunque lo llevan a cabo por cumplir con un requisito exigido por el cliente y no por transformar los procesos hacia la mejora continua y los demás beneficios internos que trae consigo la apropiación del sistema.

Para el sistema de gestión ambiental, la protección del medio ambiente y la prevención de la contaminación son preocupaciones actuales para 
cualquier organización. Según Almeida et ál. (2014), la intensificación de la conciencia pública, el debate sobre estos asuntos y las leyes nacionales y comunitarias vigentes aplicables a la gestión ambiental refuerzan la necesidad de implementar un sistema de gestión ambiental. Este es un debate que la comunidad y los sectores ambientalistas dan a la minería. Bien es cierto que los procesos de minería si no se hacen de manera adecuada llegan a impactar de manera negativa, como lo evidencia Wilson et ál. (2017); por ejemplo, el desarrollo de una mala minería en Liberia contamina el agua y genera daños irreparables en el medio ambiente, por esto, sus pobladores piden que se tomen medidas más efectivas para explotar minerales, pues muchas personas de la zona viven de esto.

Sin embargo, también se ha demostrado que con una excelente implementación de un sistema de gestión ambiental su impacto sería menor, menos degradación de la tierra, menos pérdida de biodiversidad local, menos contaminación acústica y visual. La identificación que hace Yıldız (2020), sobre la implementación de un sistema de gestión ambiental, señala que las regulaciones ambientales son demasiado estrictas y no son prácticas o aplicables, lo cual podría obstaculizar el desarrollo o causar impactos negativos para continuar con la minería. En ambos casos, no se puede alentar al desarrollo sostenible.

La gestión ambiental exitosa requiere de la coordinación adecuada de políticas, regulaciones, gestión, aplicación y organización de todos los involucrados, no solo se basa en el cumplimiento legal. Por lo tanto, una de las principales razones para implementar el sistema de gestión ambiental es preservar ambientes limpios y sostenibles, así lo mencionan Dong et ál. (2019), quienes demuestran que la industria minera puede contribuir al desarrollo sostenible y minimizar los impactos ambientales y sociales a lo largo de su ciclo de vida. El principal desafío para el sector es demostrar 
que contribuye al bienestar de la generación actual, sin comprometer la calidad de vida de las generaciones futuras.

La implementación de uno o más subsistemas de gestión en una organización requiere de un nivel mínimo de desempeño organizacional, de establecer la necesidad de sistematizar y formalizar un conjunto de procesos organizacionales relacionados con diferentes áreas de trabajo; sin embargo, según Almeida et ál. (2014), la implementación de los sistemas de gestión no siempre se realiza de manera eficaz, lo que lleva a la crítica, comúnmente referida, de que un sistema de gestión aumenta la carga de la organización y provoca una "rigidez" organizativa.

Hecha una visualización de cada sistema aplicado en el sector minero, se evidenciaron los beneficios relacionados con los problemas en los procesos organizacionales. La mejora operativa, gracias a un cambio de cultura organizacional, lleva a la reducción de los documentos, a la no duplicidad de las actividades, logra ejecutar los procesos de manera estándar, segura y responder a las necesidades y expectativas de las partes interesadas. Así, se demuestra que se puede hacer una minería bien hecha, que genere oportunidades de trabajo, mejore la calidad de vida de los trabajadores y sea sostenible con el ambiente. Articular los tres sistemas y tomar como hábito la mejora continua hará que el proceso sea más viable y menos traumáticos dentro del sector minero.

La integración es una decisión estratégica importante para promover mayores niveles de competitividad y sostenibilidad. La integración exitosa está relacionada con la verdadera motivación que lleva a las organizaciones a integrarse. Para lograr esta efectividad y un nivel de integración "todo en uno", las organizaciones deben cumplir con un conjunto de factores que conducirán al éxito y a un mejor control del 
sistema; y así abanderar el proceso en la zona carbonífera de pequeña y mediana minería del país.

El objetivo del presente artículo es identificar, desde una revisión bibliográfica, los beneficios de integrar el sistema de gestión de calidad, el sistema de gestión ambiental y el sistema de gestión de seguridad y salud en el trabajo en el sector minero, basado en la experiencia de otras compañías.

\section{Metodología}

Se desarrolló una investigación de tipo cualitativo, llevando a cabo un proceso de revisión documental en el cual se acotaron diferentes bases de datos como Science Direct, Taylor Francis Online, Springer link, Web of Sciencie y Pumbed, y los documentos tiene fecha de publicación no mayor a seis años. Se corresponde así con a una investigación descriptiva, a partir de la cual se analizan los requerimientos de contenido en términos de calidad, seguridad y gestión ambiental, aplicados al sector minero, con el fin de identificar las técnicas de implementación, sus dificultades y éxito en las organizaciones.

La revisión de los artículos científicos busca los principales beneficios de cada sistema, los factores influyentes en la implementación del sistema de gestión de calidad, seguridad y salud en el trabajo en organizaciones del sector minero; así mismo, dándose presentan parámetros de ejecución o incluso experiencias de las mismas empresas mineras, sus dificultades y el éxito que han tenido en el desarrollo de esta metodología de gestión.

Uno de los criterios para la obtención de una muestra razonable que contribuya al cumplimiento del objetivo de este estudio fue la integración de las siguientes palabras clave: "mining sector", "quality management system", "environmental management system", "occupational health and 
safety management system”. Se incluyen artículos en inglés y español, posteriormente, se realizó la aplicación de filtros de razonabilidad, principalmente de un tipo de documento: artículos de investigación. Por último, se realizó la exclusión de artículos muy específicos en cuanto a casos de estudio orientados en minería subterránea, se usaron ecuaciones de búsqueda como se muestra a continuación (ver tabla 1 y figura 1):

Tabla 1. Filtros de bases de datos

\begin{tabular}{|c|c|}
\hline Ecuación & Filtro \\
\hline $\begin{array}{c}\text { Mining without a quality management } \\
\text { system }\end{array}$ & $2014-2020$ \\
\hline $\begin{array}{c}\text { safety and health at work in mining and } \\
\text { management }\end{array}$ & $2014-2020$ \\
\hline $\begin{array}{c}\text { Environmental in mining and } \\
\text { management }\end{array}$ & $2014-2020$ \\
\hline Management system in mining & $2014-2020$ \\
\hline Fuente: elaboración propia. & \\
\hline
\end{tabular}

Signos, Investigación en Sistemas de Gestión

ISSN: 2145-1389 | e-ISSN: 2463-1140 | DOI: https://doi.org/10.15332/24631140

Vol. 13 N.o 2 | julio-diciembre de 2021 
Figura 1. Proceso de depuración de los artículos

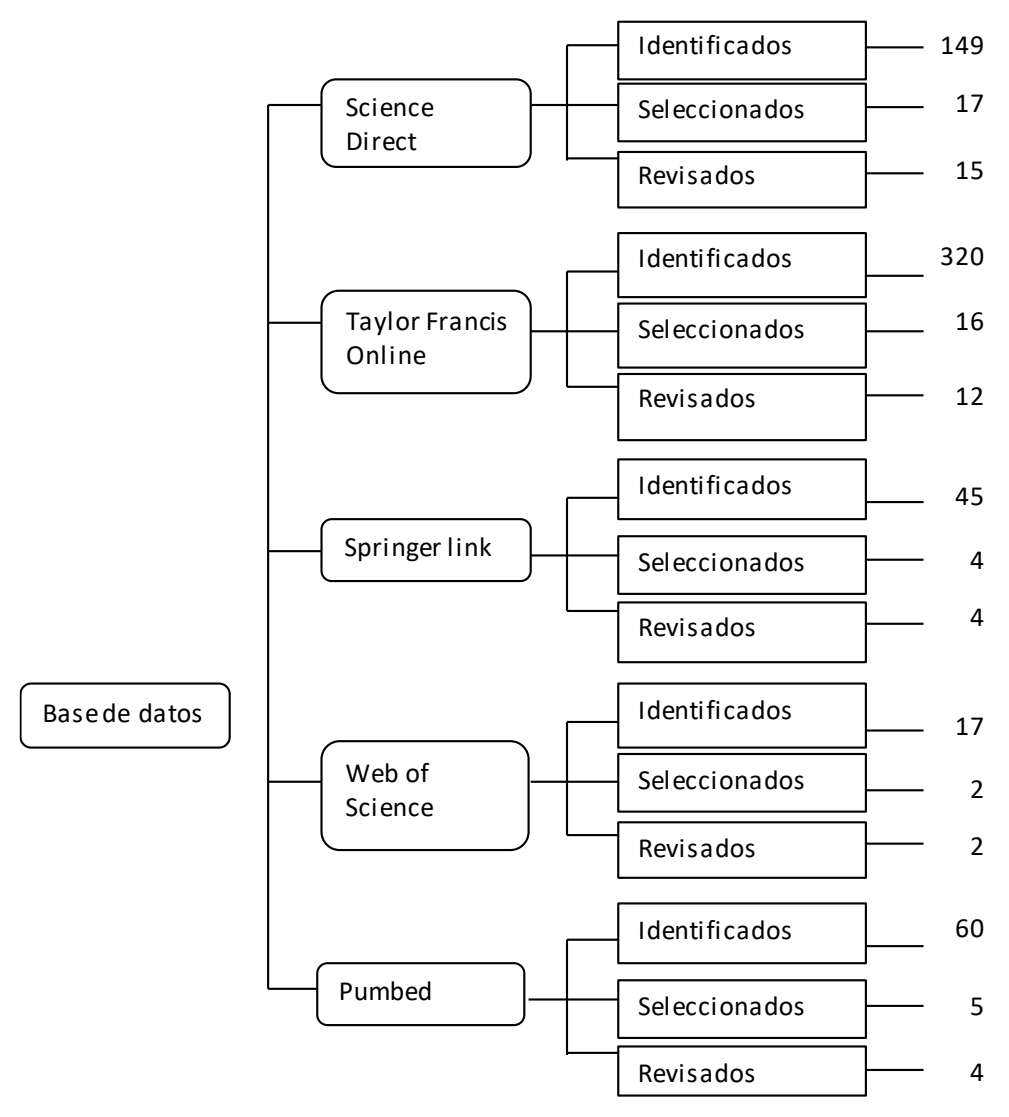

Fuente. elaboración propia.

Se identificaron 37 artículos destacados y se utilizó el resumen analítico especializado como método para el análisis de los datos recolectados, a través del cual se consideraron las siguientes variables bibliométricas: año de publicación, país e idioma, descripción de variables técnicas, conceptualización de eficiencia de los sistemas de gestión aplicados en minería, empleados para la ejecución de los temas de gestión, beneficios y limitaciones encontradas y metodologías aplicadas para el éxito del sistema de gestión.

La distribución de los 37 artículos seleccionados fue de la siguiente manera: 9 artículos sobre calidad, 13 sobre seguridad y salud en el trabajo, 14 artículos sobre gestión ambiental y 1 sobre sistema de gestión. 
Se incluyeron artículos originales, publicados en las bases datos científicas consultadas, en idioma inglés publicados el periodo comprendido entre el 2014 y 2020, los cuales cumplían con los criterios de gestión ambiental, calidad y seguridad y salud en el trabajo para el sector minero. Estos muestran los grandes retos en el sector, las problemáticas del mismo y cómo han llegado a superarlas con la implementación de sistemas integrados de gestión.

\section{Resultados y discusión}

Después del análisis del contenido de los artículos que se seleccionaron se identificaron las siguientes variables (ver tabla 2):

Tabla 2. Consolidación de resultados

\begin{tabular}{|c|c|c|c|}
\hline Variables & Dimensiones & $\begin{array}{l}\text { Número de } \\
\text { artículos } \\
\text { asociados a la } \\
\text { dimensión }\end{array}$ & Estudios de soporte \\
\hline \multirow{12}{*}{ Contexto } & \multirow{2}{*}{ Partes interesadas. } & \multirow{2}{*}{2} & Ruokonen y Temmes (2019). \\
\hline & & & Ranängen y Zobel (2014). \\
\hline & \multirow{3}{*}{$\begin{array}{l}\text { Compromiso } \\
\text { organizacional. }\end{array}$} & \multirow{3}{*}{2} & Ruokonen 2020. \\
\hline & & & $\begin{array}{l}\text { KwesiAmponsah-Tawiah 1,*, } \\
\text { Michael AkomeahOforiNtow ; } \\
\text { JusticeMensah } 2016\end{array}$ \\
\hline & & & $\begin{array}{l}\text { (Amponsah-Tawiah et al., } \\
\text { 2016). }\end{array}$ \\
\hline & \multirow{7}{*}{$\begin{array}{l}\text { Beneficios y } \\
\text { dificultades de } \\
\text { implementación. }\end{array}$} & \multirow{7}{*}{8} & $\begin{array}{l}\text { (Agencia Nacional de Mineria, } \\
\text { 2020b). }\end{array}$ \\
\hline & & & $\begin{array}{l}\text { Almeida, Domingues y Sampaio } \\
\text { (2014). }\end{array}$ \\
\hline & & & $\begin{array}{l}\text { Mežinska, Lapina y Mazais } \\
\text { (Mežinska et al., 2015). }\end{array}$ \\
\hline & & & Santos et ál. (2015). \\
\hline & & & Moumen y El Aoufir (2017). \\
\hline & & & Nunhes y Oliveira (2018). \\
\hline & & & Abisourour et ál. (2019). \\
\hline
\end{tabular}

Signos, Investigación en Sistemas de Gestión

ISSN: 2145-1389 | e-ISSN: 2463-1140 | DOI: https://doi.org/10.15332/24631140

Vol. 13 N.० 2 | julio-diciembre de 2021 


\begin{tabular}{|c|c|c|c|}
\hline Variables & Dimensiones & $\begin{array}{l}\text { Número de } \\
\text { artículos } \\
\text { asociados a la } \\
\text { dimensión }\end{array}$ & Estudios de soporte \\
\hline \multirow{21}{*}{$\begin{array}{l}\text { Método de } \\
\text { aplicación }\end{array}$} & \multirow{7}{*}{$\begin{array}{l}\text { Identificación de } \\
\text { peligros e impactos. }\end{array}$} & \multirow{7}{*}{4} & $\begin{array}{l}\text { Romero y Rodríguez-Rojas } \\
\text { (2016). }\end{array}$ \\
\hline & & & Yeoh et ál. (2015). \\
\hline & & & Liu et ál. (2020). \\
\hline & & & Samantra et ál. (2015). \\
\hline & & & $\begin{array}{c}\text { DominguesMaria S.Q. a,, } \\
\text { Baptista Adelina L.F. b, , Diogo } \\
\text { Miguel Tato a, } \Uparrow 2017\end{array}$ \\
\hline & & & (Domingues et al., 2017). \\
\hline & & & Smith et ál. (2016). \\
\hline & \multirow{8}{*}{ Cultura organizacional. } & \multirow{8}{*}{6} & Mirzaei Aliabadi et ál. (2018). \\
\hline & & & $\begin{array}{c}\text { DominguesMaria S.Q. a; } \\
\text { Baptista Adelina L.F. ; Diogo } \\
\text { Miguel Tato } 2017\end{array}$ \\
\hline & & & (Domingues et al., 2017). \\
\hline & & & $\begin{array}{l}\text { Stephen Bornstein y SusanHart } \\
2018 .\end{array}$ \\
\hline & & & (Bornstein \& Hart, 2010). \\
\hline & & & Korban (2015). \\
\hline & & & Feng et ál. (2014). \\
\hline & & & Yeoh et ál. (2015). \\
\hline & \multirow{6}{*}{ Toma de conciencia. } & \multirow{6}{*}{5} & Gallagher et ál. (2013). \\
\hline & & & Niczyporuk (2015). \\
\hline & & & Alex G. Stewart \\
\hline & & & (Stewart, 2020) \\
\hline & & & Wei et ál. (2015). \\
\hline & & & Boal (2018). \\
\hline \multirow{3}{*}{ Estrategias } & \multirow{3}{*}{$\begin{array}{l}\text { Mejoramiento } \\
\text { continuo. }\end{array}$} & \multirow{3}{*}{3} & Haas y Yorio (2016). \\
\hline & & & $\begin{array}{c}\text { Bryan Trudela,*, } \\
\text { SylvieNadeaua, } \\
\text { KazimierzZarasb and } \\
\text { IsabelleDeschampsc } 2018\end{array}$ \\
\hline & & & (Trudel et al., 2015). \\
\hline
\end{tabular}

Signos, Investigación en Sistemas de Gestión

ISSN: 2145-1389 | e-ISSN: 2463-1140 | DOI: https://doi.org/10.15332/24631140

Vol. 13 N.o 2 | julio-diciembre de 2021 


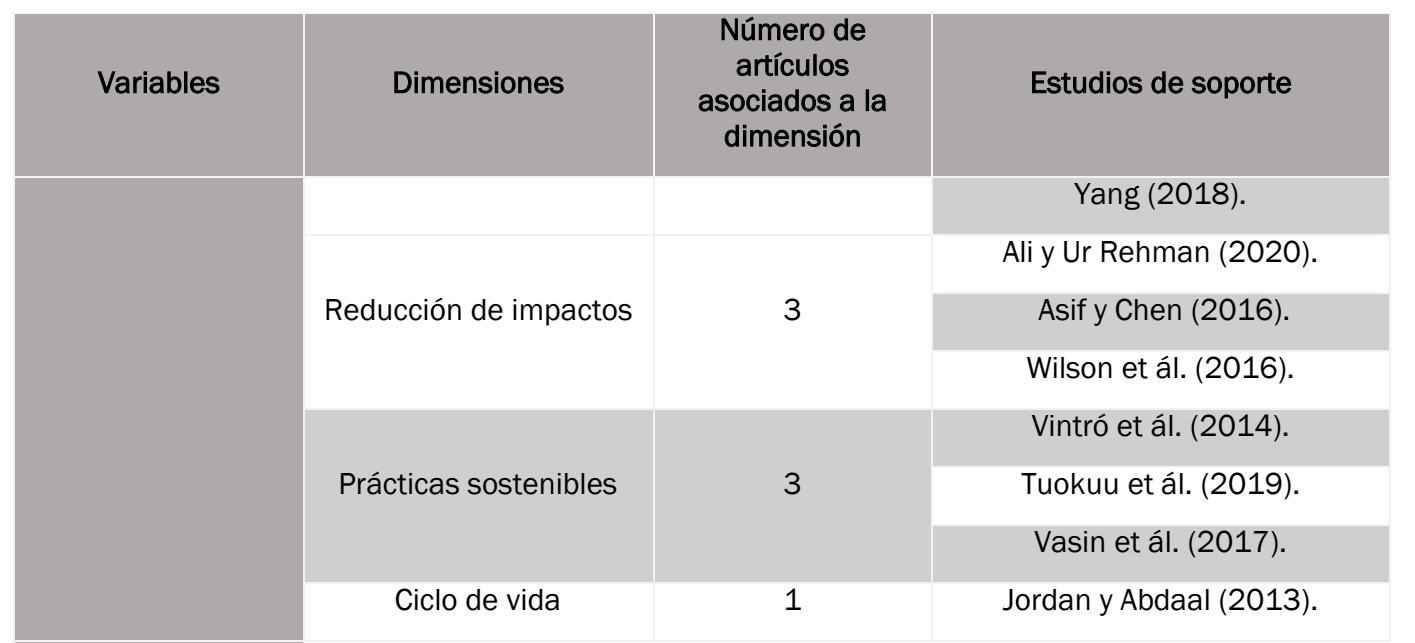

Fuente: elaboración propia.

A continuación, se hace un análisis de las variables junto con las dimensiones que las conforman:

\section{Contexto}

Dentro de esta dimensión se identificaron las siguientes variables:

Partes interesadas

Las partes interesadas representan un papel primordial dentro del desarrollo de los sistemas de gestión, se ven impactadas de manera directa o indirecta por el desarrollo de las actividades de las organizaciones. Para Ranängen y Zobel (2014) los sistemas de gestión deben complementarse con buenas prácticas de participación con los grupos identificados, ya que logran identificar y satisfacer las necesidades de cada uno y hacer que el sistema dé mejores resultados y sea sostenible. Desde la visión de Boliden (Ruokonen y Temmes, 2019) se debe tener un diálogo abierto con los grupos, ya que aportan apoyo y posicionamiento a la organización, no se puede trabajar sin ellos y son parte estratégica de la compañía. 


\section{Compromiso organizacional}

La satisfacción en el trabajo, la distribución de liderazgo o incluso la sostenibilidad de un sistema de gestión dentro de una organización no solo se mide en indicadores, sino también en la mejora de la cultura laboral, formando un compromiso en todos los niveles de la compañía. De acuerdo con Ruokonen (2020), hay factores claves para el equilibrio de un sistema de gestión como el compromiso desde la alta dirección con el sistema, establecer recursos, participar activamente en el sistema y generar canales de comunicación. Para Amponsah-Tawiah et ál. (2016), el compromiso es la fuerza que une a un individuo con un objetivo, si las organizaciones fortalecen ese vínculo hay mayor satisfacción y se logra que las personas se sientan bien haciendo las cosas, se genera confianza y participación; así mismo, es primordial que la empresa incluya el compromiso con sus trabajadores e infunda sobre ellos métodos de control, como la inspección en hábitos de seguridad que genera efectividad y control.

\section{Beneficios y dificultades de la implementación}

A partir de la revisión documentada se identifica (ver tabla 3):

Tabla 3. Identificación de beneficios y dificultades

\begin{tabular}{|c|c|c|c|}
\hline Componente & Beneficios & Dificultad & Reto \\
\hline $\begin{array}{l}\text { Compromiso por la } \\
\text { dirección. }\end{array}$ & $\begin{array}{c}\text { Compromiso con la } \\
\text { organización. } \\
\text { Asignar recursos. } \\
\text { Participación en el } \\
\text { sistema. } \\
\text { Comunicación. } \\
\text { Planificación. }\end{array}$ & $\begin{array}{l}\text { Costos de } \\
\text { implementación. } \\
\text { Falta de planificación. }\end{array}$ & $\begin{array}{l}\text { Integración de los } \\
\text { sistemas. } \\
\text { Liderazgo. } \\
\text { Garantizar recursos. }\end{array}$ \\
\hline $\begin{array}{c}\text { Identificación de } \\
\text { necesidades. }\end{array}$ & $\begin{array}{l}\text { Permite establecer el } \\
\text { alcance del sistema. } \\
\text { Genera toma de } \\
\text { decisiones. }\end{array}$ & & \\
\hline
\end{tabular}




\begin{tabular}{|c|c|c|c|}
\hline Componente & Beneficios & Dificultad & Reto \\
\hline $\begin{array}{l}\text { Participación de los } \\
\text { procesos. }\end{array}$ & $\begin{array}{l}\text { Cumplimiento de } \\
\text { requisitos. } \\
\text { Confianza. } \\
\text { Reconocimiento. } \\
\text { Involucramiento de las } \\
\text { partes interesadas. } \\
\text { Liderazgo. } \\
\text { Decisiones. } \\
\text { Administración y } \\
\text { gestión del } \\
\text { conocimiento. } \\
\text { Definiciones de } \\
\text { funciones y roles. }\end{array}$ & Resistencia al cambio. & $\begin{array}{c}\text { Aseguramiento de la } \\
\text { información por parte } \\
\text { de todos los grupos de } \\
\text { interés. } \\
\text { Mayor nivel de } \\
\text { formación de los } \\
\text { participantes del } \\
\text { sistema. }\end{array}$ \\
\hline $\begin{array}{l}\text { Identificación de } \\
\text { Información de los } \\
\text { procesos. }\end{array}$ & $\begin{array}{c}\text { Estandarización de los } \\
\text { procesos. } \\
\text { Disminuye los errores } \\
\text { en las actividades. } \\
\text { Participación. } \\
\text { Control sobre las } \\
\text { fallas. }\end{array}$ & & \\
\hline $\begin{array}{l}\text { Definición de } \\
\text { objetivos. }\end{array}$ & $\begin{array}{l}\text { Establece el horizonte } \\
\text { de la organización. }\end{array}$ & & \\
\hline
\end{tabular}

Fuente: elaboración propia.

Una vez reconocidos los principales componentes de esta dimensión, se describen a continuación de acuerdo con la orientación de los autores consultados.

Toda compañía en el desarrollo de la implementación de un sistema de gestión busca obtener los beneficios, tanto externos como internos. De acuerdo con Romero y Rodríguez-Rojas (2016), uno de los principales provechos es mejorar los procesos de la organización y ser más competitiva y así responder a las necesidades del mercado. Para Almeida et ál. (2014) los beneficios se reflejan en el cambio estructural de la organización, la reducción de documentos, mejorar la comunicación, liderazgo, entendimiento del sistema; se resalta que la motivación y 
participación de los empleados son esenciales ya que llegan a ser responsables de una parte del proceso.

Se identifica que una estructura definida en roles y autoridades ayuda en la apropiación del sistema para el personal. Como lo menciona Moumen y El Aoufir (2017) lograr una simplificación del mismo hace que las personas asuman su participación en el desarrollo de las acciones, genera menos reproceso de las actividades y sí un cumplimento legal óptimo que logra dar respuesta a los entes de control, clientes, Gobierno y demás partes interesadas.

Para algunas organizaciones se presentan dificultades en el transcurso de la implementación e integración del sistema de gestión. De acuerdo con Santos et ál. (2016), los beneficios no superan los costos de implantación de las primeras etapas, su inversión no solo es el cambio estructural, si no cultural, la mayoría de las empresas implementan un sistema para marketing y negocios y no ven muy claro el papel del sistema de gestión dentro de la compañía. Dentro de las primeras etapas de implantación se evidencia la resistencia al cambio, los trabajadores no se adaptan, en este proceso es considerable infundir la participación y comunicación de ellos motivándolos al desarrollo de la mejora continua. Nunhes y Oliveira (2020) identifican que si un sistema es seguro los trabajadores confían, participan, logran ser eficientes desde sus puestos de trabajo, dando una mejor compatibilidad y sinergia en el entorno. Es un trabajo largo, pero cuando se fijan objetivos estratégicos y alcanzables es notable la reducción de costos, accidentalidad y la disminución de impactos en el ambiente. Más allá de lograr identificar problemas operativos como lo aluden Abisourour et él. (2020), la reducción del tiempo y la limitación de pérdidas son resultados que se ven a mediano plazo de la implementación de cualquier sistema de gestión y a su vez logra la satisfacción de las 
necesidades de las partes interesadas. También se observa que la toma decisiones, basada en las dificultades dadas en el proceso, logran ser más asertivos, generar menos costos y, sobre todo, da a la compañía experiencia, pues un error no se comete dos veces.

De acuerdo con lo anterior, son más los beneficios que la dificultad en el desarrollo de un sistema de gestión, actualmente una organización que no esté con un sistema tiende a desaparecer.

\section{Método de aplicación}

El método dentro de una etapa es crucial, ya que a través de este se garantiza la correcta realización de los procesos y se asegura el cumplimento de los objetivos trazados dentro del sistema de gestión. A partir de este punto, se identifican las dimensiones dadas como resultado de la revisión bibliográfica (ver figura 2):

Figura 2. Resultado de la variable método

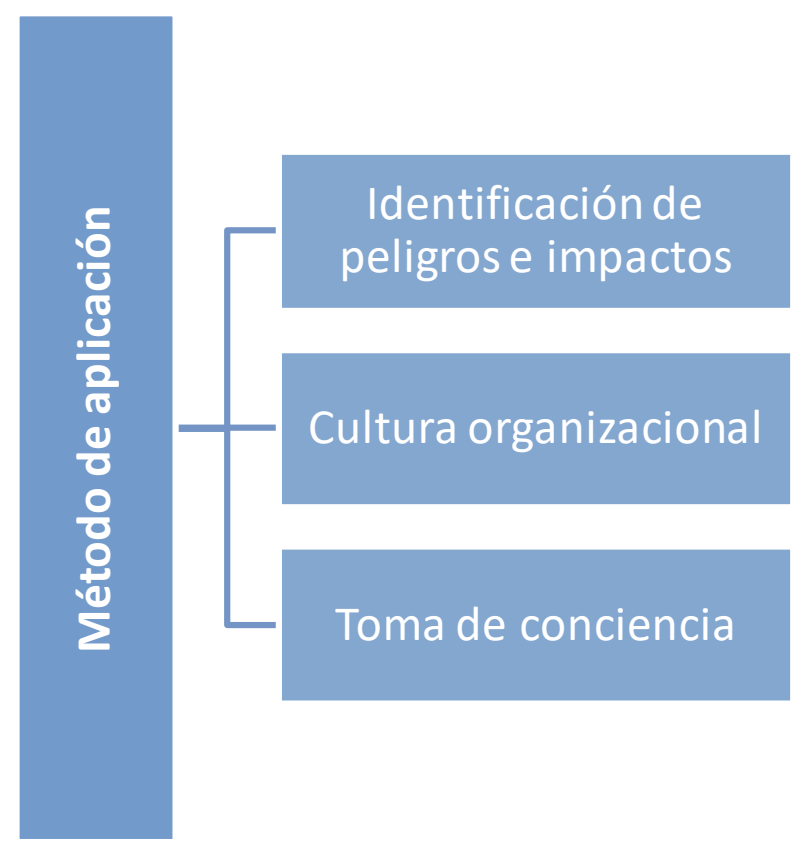

Fuente: elaboración propia. 


\section{Identificación de peligros e impactos}

El enfoque de la identificación de peligros dentro del desarrollo de los procesos logra la seguridad dentro del sistema; se encontró que este aumenta los índices de calidad del trabajo y confianza en el trabajador para desarrollar sus labores, se identificó también que el proceso de autoconciencia frente a un riesgo, si no se trabaja continuamente, eleva el índice de accidentalidad en la minería subterránea. De acuerdo con Liu et ál. (2020) es fundamental determinar los riesgos para un control efectivo, ya que ayuda a una correcta toma de decisiones y aun adecuado seguimiento, con el fin de evitar que se repitan las causas y se generen accidentes nuevos. No desarrollar un sistema de gestión en seguridad aumenta notablemente los eventos no controlables y se expone a pérdida de vidas, recursos económicos y desconfianza antes los clientes (Domingues et ál., 2017).

Dentro de la identificación de aspectos ambientales, se aseguró que el objetivo es la reducción de su impacto con el fin de establecer estrategias de sostenibilidad a partir del enfoque de ciclo de vida. Smith et ál. (2016) mencionan que lograr un análisis de los impactos desde el principio hasta el fin del proceso ayuda a identificar y controlar todo lo que puede impactar de manera negativa al sistema. Como se mencionaba anteriormente, tomar decisiones acertadas y fijar intervenciones asegura ambientes positivos, genera un control de las emisiones contaminantes y protege el ambiente (Samantra et ál, 2017).

\section{Cultura organizacional}

Dentro de los sistemas de gestión se observa la articulación de los procesos con las estrategias trazadas por la compañía y la forma como esta interactúa en conjunto. De acuerdo con Stemn et ál. (2019), el fortalecimiento de la cultura logra un desempeño dentro del desarrollo de 
las tareas, la reducción del reproceso y los productos no conformes que ayudan a un mejor resultado. Como lo identifica Mirzaei Aliabadi et ál. (2018), la presión organizacional y la falta de conocimiento resultan en el desarrollo incorrecto de las actividades y en que se asuman percepciones diferentes frente a su desarrollo. Es importante crear un clima idóneo que dé equilibrio entre todos y ayudar a mantenerlo para favorecer el entorno, generar un factor de confianza y estabilidad en la organización, representado en una alineación entre lo financiero, el cliente, los procesos internos y de aprendizaje, todo con el fin de establecer direcciones operativas y tácticas, para analizar la información y lograr una toma de decisiones asertivas. Se evidencia que el compromiso y la capacitación de las personas adopta de manera positiva la transición de los procesos, la orientación del aprendizaje ayuda a la mejora continua, al desempeño de la organización y a la adaptación a nuevos sistemas que puedan darle más prestigio y competitividad a la empresa (Feng et ál., 2014).

Para Yeoh et él. (2014), la cultura se considera un medio importante para garantizar un buen desempeño en materia de seguridad; por ejemplo, para la minería es primordial establecer este criterio, pues las condiciones son adversas y convivir con un riesgo puede cobrar la vida misma del minero. La industria minera, de acuerdo con Domingues et ál. (2017), es una técnica compleja en la cual se involucran factores humanos, organizacionales y tecnológicos, y en el cual la incertidumbre y el comportamiento humano están presentes. Por esto se debe trabajar en una concientización y compromiso con la vida, es claro que la seguridad, como cualquier otro sistema de gestión, es tan importante como la producción (Korban, 2015). Por esto se debe laborar de manera conjunta para que los resultados sean positivos y se logre llegar al mejoramiento continuo de todos los niveles de la empresa. 


\section{Toma de conciencia}

La toma de conciencia es el empoderamiento de las personas en la organización del desarrollo de las actividades del sistema de gestión y la articulación de manera efectiva del mismo para el logro de objetivos. En el caso del sector minero, es una dimensión que se debe trabajar fuertemente, Wei et ál. (2016) definieron que la falta de capacitación logra influir en las percepciones y comportamientos de los individuos.

Algunas organizaciones han asumido los sistemas de gestión como instrumentos para el desarrollo de toma de conciencia y lograr un cambio en la cultura organizacional. De acuerdo con Gallagher et ál. (2003) debe existir una estrategia de comunicación con todos los niveles de la compañía, el compromiso de la alta dirección, la definición de funciones y responsabilidades, entre otras. Para Niczyporuk (2015) el aumento de empeño de los trabajadores con el sistema logra el éxito de las metas trazadas por la organización, pero se debe apropiar de manera positiva.

Para el sector minero es importante que los mineros sean prevencionistas en preservar la salud, ya que los riesgos son inminentes en esta ardua labor. Acorde con la investigación de Stewa (2019), se encontró que el crecimiento de los índices de enfermedades laborales se está convirtiendo en un problema social y que se está deteriorando la salud de los mineros a largo plazo. El mismo estudio afirma que la mejor inversión es disminuir el riesgo y aumentar la prevención en todos sus niveles. Las empresas deben idear métodos o estrategias que logren infundir la importancia del auto cuidado en la población trabajadora.

Se debe establecer una metodología organizada, cicla, participativa que logre cubrir las necesidades de todas las partes interesadas, pues los trabajadores hacen parte de este grupo. Como lo mencionó Vintró et ál. (2014), de acuerdo a la experiencia de varias organizaciones, la 
comprensión de los aspectos positivos y negativos de las tareas diarias hace que madure la organización e implemente prácticas acordes con la población trabajadora y el favorecimiento del sistema.

\section{Estrategias}

Dentro del análisis de información, ubicamos las siguientes dimisiones como estrategias que apoyan al desarrollo de un sistema de gestión (ver figura 3):

Figura 3. Resultado de la variable estrategias

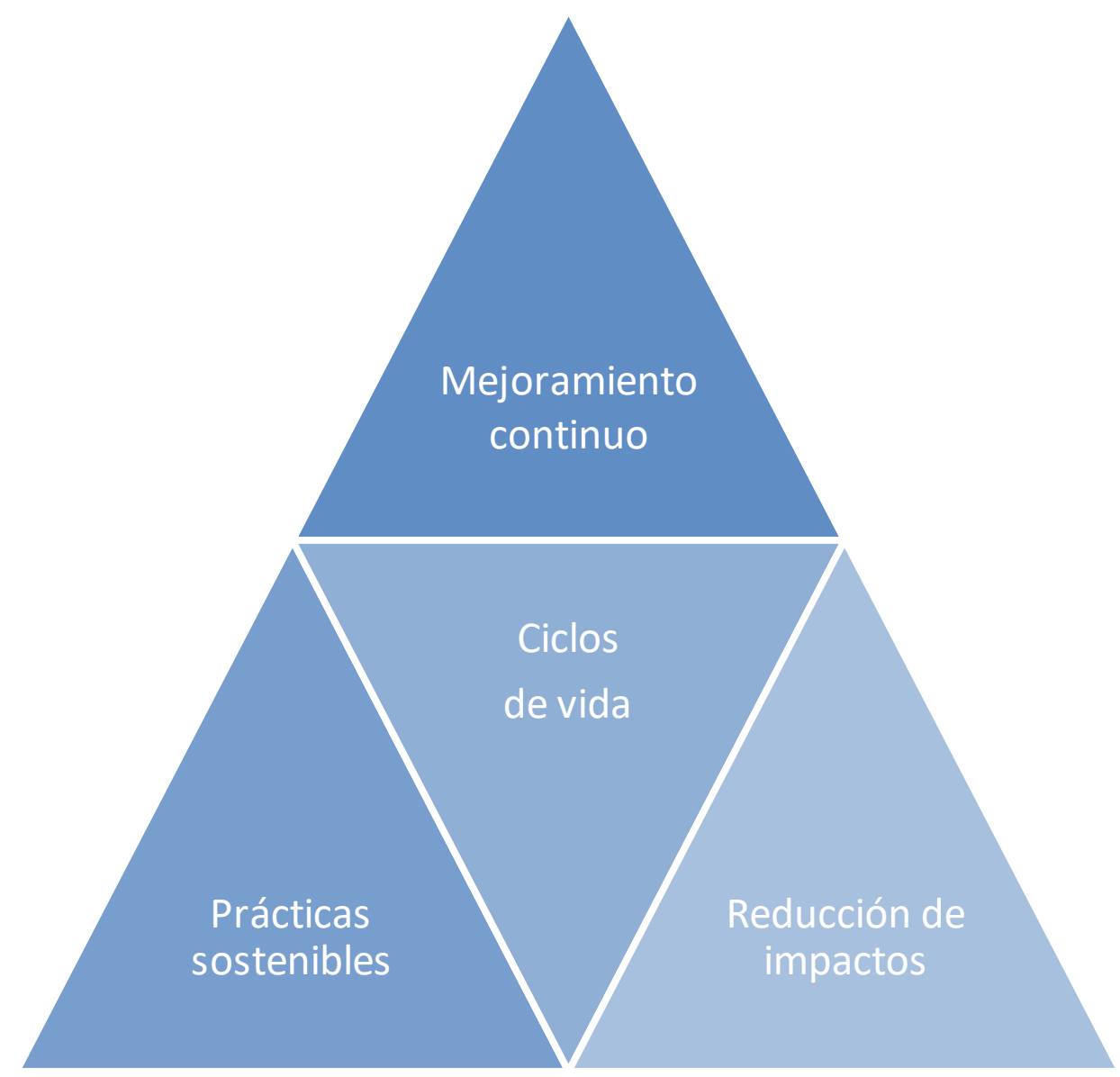

Fuente: elaboración propia. 


\section{Mejoramiento continúo}

El desarrollo de los sistemas de gestión y la mejora continua permiten que los procesos sean enfocados bajo los objetivos trazados por la organización. De acuerdo con la revisión de la documentación se identifican factores importantes en el mejoramiento continuo (ver figura 4):

Figura 4. Factores del mejoramiento continuo

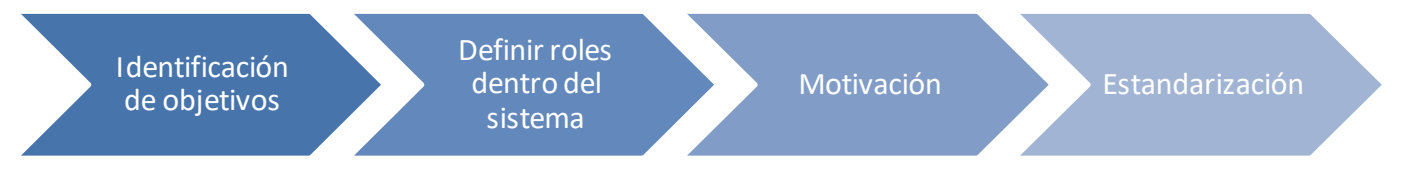

Fuente: elaboración propia.

Para un mejoramiento oportuno y cíclico, se deben establecer objetivos y metas con el fin de verificar el correcto desarrollo de las actividades (Trudel et ál., 2015), ya que logra medir el cumplimiento y establece el rumbo de la organización, a su vez, definir roles y niveles de autoridad identifica la participación de todos y ayuda a la implementación y sostenimiento del sistema desde el inicio de las operaciones.

Para el sector minero la eficacia y el mejoramiento continuo se basa en el comportamiento, la capacidad de que los mineros logren trabajar de manera segura y motiven a otros hacerlo. De acuerdo con Haas y Yorio (2016) esto ayuda a crear una cultura de prevención, mejorar la estandarización de procedimientos de trabajo y a reforzar la competencia de los trabajadores. Una buena implementación, como lo define Yang (2020), puede darse por etapas de manera progresiva con el fin de fortalecer el sistema y que se abarquen todas las áreas.

\section{Reducción de impactos}

Para el sistema de gestión ambiental es primordial la disminución de impactos dentro de los procesos, identificar el ciclo de vida ayuda al 
control de las actividades y los procesos, así se logra una producción más limpia y una estabilidad con el medio ambiente hacia la reducción de impactos (Wilson et ál., 2017; Asif y Chen, 2016; Ali y Ur Rehman, 2020).

Los autores resaltan que la base para la reducción de impactos es una buena identificación del ciclo de vida en todos los procesos; esto logra resultados que aportan al cumplimiento de los objetivos y metas de la compañía y los posibles horizontes que se pueden llegar a enfrentar, todo esto da como resultado una toma de decisiones acertadas.

\section{Prácticas sostenibles}

Estas acciones, encaminadas al mejoramiento de las actividades internas de un proceso o sistema de gestión ambiental, pretenden reducir los impactos. De acuerdo con Vintró et ál. (2014) las empresas deben establecer mecanismos para identificar, medir y controlar sus efectos ambientales. Dentro de las prácticas más usuales para esto están el definir políticas, objetivos y documentar procedimientos, esto ayuda a la estandarización de procesos y la apropiación del sistema. De acuerdo con Tuokuu et ál. (2019), las mejores prácticas relacionadas son la gobernanza, la participación de las partes interesadas y las prácticas ambientales con políticas, todas ayudan a que haya un equilibrio entre el ambiente y su desarrollo.

Otra variable que ayuda a la realización de prácticas sostenibles es la participación de la alta dirección, ya que genera motivación del recurso humano. La disponibilidad de recursos financieros para el desarrollo del sistema facilita la ejecución de actividades y genera resultados que logran ser positivos. De acuerdo con Tuokuu et ál. (2019), todo esto se adopta como decisiones que apoyan la elaboración y mejora del sistema. Establecer un método de inspección logra identificar aspectos positivos y negativos en el desarrollo de las actividades y es una herramienta para 
tomar medidas asertivas. Vasin et ál. (2017) menciona que familiarizar a las personas con la visualización de las actividades logra percibir fallas y con ello minimizar los errores en los lugares de trabajo, consigue aumentar la productividad, dando resultados más oportunos, con las medidas tomadas dentro del proceso y no al final.

\section{Ciclo de vida}

Es comprender cómo inician y terminan los procesos, al identificar aspectos y controles que logren la adaptabilidad del sistema. En la minería requieren metodologías de identificación para abordar la complejidad de los problemas ambientales y de la contaminación relacionada con las actividades mineras (Jordan y Abdaal, 2013), para identificar a lo largo del proceso las pautas que intervienen en el ciclo de vida y que ayudarían a planificar las acciones con el fin de poder anticiparse a los hechos que puedan salir de control. Esto daría una visión de cómo enfrentar las situaciones.

Dentro del desarrollo de los ciclos de vida, como lo menciona AmponsahTawiah et ál. (2016), el compromiso organizacional y la influencia de un cambio de cultura logra el cumplimiento de los objetivos propuestos, el aseguramiento de un eficiente ciclo implica un aumento de costos en certificación y un mayor esfuerzo en la participación del personal, ya que la toma de conciencia (Vintró et ál., 2014) logra que se apropie la información y ayuda a la estandarización, haciendo que la planificación funcione, que se logre una adaptación eficiente en la organización y un mayor facilidad hacia la transición al cambio.

\section{Conclusiones}

Los principales beneficios asociados a la integración de sistemas en el sector minero se orientan a la identificación de las necesidades y 
expectativas de las partes interesadas. La participación y el compromiso de la alta gerencia junto con el compromiso de quienes conforman la organización impactan de forma positiva hacia la creación de hábitos que, a futuro, consolidarán una cultura de la gestión integral. Gestionar desde el enfoque PHVA también se convierte en un elemento clave de éxito para las compañías del sector.

El enfoque a procesos es otro de los principales beneficios que perciben las organizaciones del sector, el cual permite organizar las actividades, hacer seguimiento, toma de decisiones basado en evidencias, así como el establecimiento de roles y responsabilidades, que aseguren las laborales al interior de la organización.

La implementación de un sistema de gestión dentro de las organizaciones trae beneficios económicos, estructurales y culturales, se logra así perfeccionar los procesos al maximizar la eficiencia y reducir los errores y mejorar la coordinación de los objetivos.

De acuerdo con la búsqueda, se evidencia que la aplicación en el sector minero es muy viable, permite reducir la accidentalidad, los impactos ambientales, mejorar los procesos internos de la organización y documentar toda la operación. Se logra involucrar los grupos de interés al apropiarse del sistema y persiguiendo los resultados.

Organizar y estandarizar los procesos agiliza y simplifica la información, genera una toma de decisiones correctas y elimina potenciales riesgos. Las empresas de mediana y pequeña minería lograrían mejorar sus métodos de organización para obtener resultados positivos a mediano y largo plazo. Integrar un sistema de gestión de calidad, el sistema de gestión ambiental y el sistema de gestión de seguridad y salud en el trabajo en el sector minero, de acuerdo con la revisión bibliográfica realizada, demuestra que mejora de manera continua la calidad de sus procesos, incrementa la 
confianza, la participación y la comunicación de todos los trabajadores. A su vez se evidencia la disminución del riesgo, ya que se trabaja de forma coordinada con todos los procesos y actividades de la organización.

Se identificó que la alta dirección como pilar del liderazgo y compromiso frente a cualquier sistema de gestión, posibilita los cambios positivos para todos los procesos; así genera compromiso, participación, recursos económicos, humanos, tecnológicos y comunicación desde todos los niveles, es una gran influencia en la aceptación de los cambios de implementación.

\section{Referencias}

Abisourour, J., Hachkar, M., Mounir, B., y Farchi, A. (2020). Methodology for integrated management system improvement: combining costs deployment and value stream mapping. International Journal of Production Research, 58(12), 3667-3685. https://doi.org/10.1080/00207543.2019.1633482

Amponsah-Tawiah, K., y Mensah, J. (2016). Occupational Health and Safety and Organizational Commitment: Evidence from the Ghanaian Mining Industry. Safety and Health at Work, 7(3), 225-230. https://doi.org/10.1016/j.shaw.2016.01.002

Agencia Nacional de Minería. (2020a). Así es nuestra Colombia minera. Agencia Nacional de Minería. https://www.anm.gov.co/?q=emergencias mineras

Agencia Nacional de Minería. (2020b, junio 30). Emergencias Mineras. Agencia Nacional de Mineria. https://www.anm.gov.co/?q=emergencias mineras

Ali, D., y Ur Rehman, A. (2020). Adoption of autonomous mining system in Pakistan Policy, skillset, awareness and preparedness of stakeholders. Resources Policy, 68, 101796. https://doi.org/10.1016/j.resourpol.2020.101796

Almeida, J., Domingues, P., y Sampaio, P. (2014). Different perspectives on management systems integration. Total Quality Management \& Business Excellence, 25(3-4), 338-351. https://doi.org/10.1080/14783363.2013.867098 
Amponsah-Tawiah, K., Ntow, M. A. O., y Mensah, J. (2016). Occupational Health and Safety Management and Turnover Intention in the Ghanaian Mining Sector. Safety and Health at Work, 7(1), 12-17. https://doi.org/10.1016/j.shaw.2015.08.002

Asif, Z., y Chen, Z. (2016). Environmental management in North American mining sector. Environmental Science and Pollution Research, 23, 167-179. https://link.springer.com/article/10.1007/s11356-015-5651-8

Bayram, M. (2020). Factors affecting employee safety productivity: An empirical study in an OHSAS 18001-certified organization. International Journal of Occupational Safety and Ergonomics, 1-14. https://doi.org/10.1080/10803548.2020.1739892

Boal, W. M. (2018). Work intensity and worker safety in early twentieth-century coal mining. Explorations in Economic History, 70, 132-149. https://doi.org/10.1016/j.eeh.2018.08.001

Bornstein, S., y Hart, S. (2010). Evaluating Occupational Safety and Health Management Systems: A Collaborative Approach. Policy and Practice in Health and Safety, 8(1), 61-76. https://doi.org/10.1080/14774003.2010.11667742

Chen, J. K. C., y Zorigt, D. (2013). Managing occupational health and safety in the mining industry. Journal of Business Research, 66(11), 2321-2331. https://doi.org/10.1016/j.jbusres.2012.04.013

Decreto 1072 de 2015 (26 de mayo), por medio del cual se expide el Decreto Único Reglamentario del Sector Trabajo. Diario oficial 49 523. http://www.suinjuriscol.gov.co/viewDocument.asp?ruta=Decretos $/ 30019522$

Decreto 1886 de 2015 (21 de septiembre), Por el cual se establece el Reglamento de Seguridad en las Labores Mineras Subterráneas. Diario oficial 49642. https://www.funcionpublica.gov.co/eva/gestornormativo/norma.php?i=65325

Domingues, J. P. T., Sampaio, P., y Arezes, P. M. (2017). Analysis of certified occupational health and safety management systems in Portugal. International Journal of Occupational and Environmental Safety, 1(1), 11-28.

https://doi.org/10.24840/2184-0954001.0010002

Domingues, M., Baptista, A., y Diogo, M. (2017). Engineering complex systems applied to risk management in the mining industry. International Journal of Mining Science and Technology, 27(4), 611-616. https://doi.org/10.1016/j.ijmst.2017.05.007

Signos, Investigación en Sistemas de Gestión

ISSN: 2145-1389 | e-ISSN: 2463-1140 | DOI: https://doi.org/10.15332/24631140

Vol. 13 N. ${ }^{\circ} 2$ | julio-diciembre de 2021 
Dong, L., Tong, X., Li, X., Zhou, J., Wang, S., y Liu, B. (2019). Some developments and new insights of environmental problems and deep mining strategy for cleaner production in mines. Journal of Cleaner Production, 210, 1562-1578.

https://doi.org/10.1016/j.jclepro.2018.10.291

Feng, T., Zhao, G., y Su, K. (2014). The fit between environmental management systems and organisational learning orientation. International Journal of Production Research, 52(10), 2901-2914. https://doi.org/10.1080/00207543.2013.857055

Gallagher, C., Underhill, E., y Rimmer, M. (2003). Occupational safety and health management systems in Australia: Barriers to success. Policy and Practice in Health and Safety, 1(2), 67-81. https://doi.org/10.1080/14774003.2003.11667637

Haas, E. J., y Yorio, P. (2016). Exploring the state of health and safety management system performance measurement in mining organizations. Safety Science, 83, 4858. https://doi.org/10.1016/j.ssci.2015.11.009

Icontec. (2010). Guía técnica colombiana GTC 45. Guía para la identificación de los peligros y la valoración de los riesgos en seguridad y salud ocupacional. Icontec.

Icontec. (2015a). Norma Técnica Colombiana NTC-ISO 9001. Sistema de Gestión de la Calidad, Requisitos. Icontec.

Icontec. (2015b). Norma Técnica Colombiana NTC-ISO 14OO1. Sistema de Gestión Ambiental, Requisitos. Icontec.

Icontec. (2018). Norma Técnica Colombiana NTC-ISO 45001. Sistema de Gestión de Seguridad y Salud en el trabajo. Requisitos. Icontec.

Jordan, G., y Abdaal, A. (2013). Decision support methods for the environmental assessment of contamination at mining sites. Environmental Monitoring and Assessment, 185(9), 7809-7832. https://doi.org/10.1007/s10661-013-3137-z

Korban, Z. (2015). Quality assessment of occupational health and safety management at the level of business units making up the organizational structure of a coal mine: a case study. International Journal of Occupational Safety and Ergonomics, 21(3), 373-381. https://doi.org/10.1080/10803548.2015.1081776

Liu, Q., Dou, F., y Meng, X. (2020). Building risk precontrol management systems for safety in China's underground coal mines. Resources Policy, 101631. https://doi.org/10.1016/j.resourpol.2020.101631 
Marchiori, D., y Mendes, L. (2020). Knowledge management and total quality management: Foundations, intellectual structures, insights regarding evolution of the literature. Total Quality Management \& Business Excellence, 31(9-10), 11351169. https://doi.org/10.1080/14783363.2018.1468247

Mirzaei Aliabadi, M., Aghaei, H., Kalatpour, O., Soltanian, A. R., y Seyed Tabib, M. (2018). Effects of human and organizational deficiencies on workers' safety behavior at a mining site in Iran. Epidemiology and Health, 4O, e2018019. https://doi.org/10.4178/epih.e2018019

Mežinska, I., Lapiṇa, I., y Mazais, J. (2015). Integrated management systems towards sustainable and socially responsible organisation. Total Quality Management \& Business Excellence, 26(5-6), 469-481. https://doi.org/10.1080/14783363.2013.83589

Moumen, M., y El Aoufir, H. (2017). Quality, safety and environment management systems (QSE): Analysis of empirical studies on integrated management systems (IMS). Journal of Decision Systems, 26(3), 207-228. https://doi.org/10.1080/12460125.2017.1305648

Niczyporuk, Z. T. (2015). Safety Management in Coal Mines-Risk Assessment. International Journal of Occupational Safety and Ergonomics, 2(3), 243-250. https://doi.org/10.1080/10803548.1996.11076352

Nikulin, A., y Nikulina, A. (2017). Assessment of occupational health and safety effectiveness at a mining company. Ecology, Environment and Conservation, 23(1), 351-355. https://www.researchgate.net/publication/317820768 Assessment of occupatio nal health and safety effectiveness at a mining company

Nunhes, T. V., y Oliveira, O. J. (2020). Analysis of Integrated Management Systems research: Identifying core themes and trends for future studies. Total Quality Management \& Business Excellence, 31(11-12), 1243-1265. https://doi.org/10.1080/14783363.2018.1471981

Pantoja Timarán, F. H., y Pantoja Barrios, S. D. (2016). Problemas y desafíos de la minería de oro artesanal y en pequeña escala en Colombia. Revista Facultad de Ciencias Económicas, 24(2). https://doi.org/10.18359/rfce.2217

Signos, Investigación en Sistemas de Gestión ISSN: 2145-1389 | e-ISSN: 2463-1140 | DOI: https://doi.org/10.15332/24631140 Vol. 13 N.o 2 | julio-diciembre de 2021 
Pink, S., Waterson, P., Dainty, A., Cheyne, A., Haslam, R., Gibb, A., Morgan, J., Hartley, R., Finneran, A., y Bust, P. (2016). Interdisciplinary research for occupational safety and health knowledge. Policy and Practice in Health and Safety, 14(1), 2233. https://doi.org/10.1080/14773996.2016.1235832

Ranängen, H., y Zobel, T. (2014). Exploring the path from management systems to stake holder management in the Swedish mining industry. Journal of Cleaner Production, 84, 128-141. https://doi.org/10.1016/j.jclepro.2014.04.025

Romero, C. C., y Rodríguez-Rojas, Y. L. (2016). Beneficios e impactos de la implementación de normas técnicas en las organizaciones: Una revisión sistemática. Signos, Investigación en Sistemas de Gestión, 8(2), 133-167. https://doi.org/10.15332/s2145-1389.2016.0002.07

Ruokonen, E. (2020). Preconditions for successful implementation of the Finnish standard for sustainable mining. The Extractive Industries and Society, 7(2), 611620. https://doi.org/10.1016/j.exis.2020.03.008

Ruokonen, E., y Temmes, A. (2019). The approaches of strategic environmental management used by mining companies in Finland. Journal of Cleaner Production, 210, 466-476. https://doi.org/10.1016/j.jclepro.2018.10.273

Sadeghi Moghadam, M. R., Safari, H., y Yousefi, N. (2019). Clustering quality management models and methods: Systematic literature review and text-mining analysis approach. Total Quality Management \& Business Excellence, 33(3-4), 124. https://doi.org/10.1080/14783363.2018.1540927

Samantra, C., Datta, S., y Mahapatra, S. (2017). Analysis of occupational health hazards and associated risks in fuzzy environment: A case research in an Indian underground coal mine. International Journal of Injury Control and Safety Promotion, 24(3), 311-327. https://doi.org/10.1080/17457300.2016.1178298

Santos, G., Rebelo, M., Lopes, N., Alves, M. R., y Silva, R. (2016). Implementing and certifying ISO 14001 in Portugal: Motives, difficulties and benefits after ISO 9001 certification. Total Quality Management \& Business Excellence, 27(11-12), 12111223. https://doi.org/10.1080/14783363.2015.1065176

Signos, Investigación en Sistemas de Gestión

ISSN: 2145-1389 | e-ISSN: 2463-1140 | DOI: https://doi.org/10.15332/24631140

Vol. 13 N.o 2 | julio-diciembre de 2021 
Smith, N. M., Ali, S., Bofinger, C., y Collins, N. (2016). Human health and safety in artisanal and small-scale mining: An integrated approach to risk mitigation. Journal of Cleaner Production, 129, 43-52. https://doi.org/10.1016/j.jclepro.2016.04.124

Stemn, E., Bofinger, Cliff, y Hassall. (2019). Examining the relationship between safety culture maturity and safety performance of the mining industry. Safety Science, 113, 345-355. https://doi.org/10.1016/j.ssci.2018.12.008

Stewart, A. (2020). Mining is bad for health: A voyage of discovery. Environmental Geochemistry and Health, 42, 1153-1165. https://doi.org/10.1007/s10653-019$\underline{00367-7}$

Trudel, B., Nadeau, S., Zaras, K., y Deschamps, I. (2015). Managing equipment innovations in mining: A review. Work, 51(4), 731-746. https://doi.org/10.3233/WOR-152033

Tuokuu, F. X. D., Idemudia, U., Gruber, J. S., y Kayira, J. (2019). Identifying and clarifying environmental policy best practices for the mining industry-A systematic review. Journal of Cleaner Production, 222, 922-933. https://doi.org/10.1016/j.jclepro.2019.03.111

Vanek, M., Spakovska, M. y Pomothy, L. (2015). Continuous improvement management for mining companies. Journal of the Southern African Institute of Mining and Metallurgy, 115(2), 119-124. http://www.scielo.org.za/pdf/jsaimm/v115n2/o7.pdf

Vasin, A. A., Kozyreva, E. A., y Tyuleneva, A. S. (2017). Optimization of the occupational safety compliance control (OSCC) system at a coal mining enterprise. Journal of Computer and Systems Sciences International, 56(5), 810-817. https://doi.org/10.1134/S1064230717050124

Vintró, C., Sanmiquel, L., y Freijo, M. (2014). Environmental sustainability in the mining sector: Evidence from Catalan companies. Journal of Cleaner Production, 84, 155163. https://doi.org/10.1016/j.jclepro.2013.12.069

Wei, J., Cheng, P., y Zhou, L. (2016). The Effectiveness of Chinese Regulations on Occupational Health and Safety: A Case Study on China's Coal Mine Industry. Journal of Contemporary China, 25(102), 923-937. https://doi.org/10.1080/10670564.2016.1186369 
Wilson, S. T. K., Wang, H., Kabenge, M., y Qi, X. (2017). The mining sector of Liberia: Current practices and environmental challenges. Environmental Science and Pollution Research, 24(23), 18711-18720. https://doi.org/10.1007/s11356-0179647-4

Yang, C.-C. (2020). The effectiveness analysis of the practices in five quality management stages for SMEs. Total Quality Management \& Business Excellence, 31(9-10), 955977. https://doi.org/10.1080/14783363.2018.1456010

Yeoh, W., Richards, G., y Wang, S. (2014). Benefits and Barriers to Corporate Performance Management Systems. Journal of Computer Information Systems, 55(1), 105-116. https://doi.org/10.1080/08874417.2014.11645745

Ylldız, T. D. (2020). The impacts of EIA procedure on the mining sector in the permit process of mining operating activities \& Turkey analysis. Resources Policy, 67, 101681. https://doi.org/10.1016/j.resourpol.2020.101681 